\title{
A Neural Basis for Percept Stabilization in Binocular Rivalry
}

\author{
Philipp Sterzer and Geraint Rees
}

\begin{abstract}
When the same visual input has conflicting interpretations, conscious perception can alternate spontaneously between each competing percept. Surprisingly, such bistable perception can be stabilized by intermittent stimulus removal, suggesting the existence of perceptual "memory" across interruptions in stimulation. The neural basis of such a process remains unknown. Here, we studied binocular rivalry, one type of bistable perception, in two linked experiments in human participants. First, we showed, in a behavioral experiment using binocular rivalry between face and grating stimuli, that the stabilizing effect of stimulus removal was specific to perceptual alternations evoked by rivalry, and did not occur following physical alternations in the absence of rivalry. We then used functional
\end{abstract}

\section{INTRODUCTION}

Binocular rivalry is one example of a more general family of bistable perceptual phenomena. When dissimilar images are presented to the two eyes, they compete for perceptual dominance with each image being visible for a few seconds while the other is suppressed. Conscious awareness thus alternates between two different interpretations of the same visual input. It has been widely believed that this alternation is the automatic and inevitable competitive outcome of viewing stimuli without a unique solution (Blake \& Logothetis, 2002). However, temporary removal of bistable visual stimuli can slow, and even stop, alternations, stabilizing perception in one of the two possible states (Leopold, Wilke, Maier, \& Logothetis, 2002). Specifically, when bistable stimuli are made to disappear for several seconds, the percept on reappearance tends to be the same as before they disappeared. This survival of a conscious perceptual state across such a delay period of many seconds is involuntary, and suggests the presence of an unexpected and persistent perceptual memory associated with such percept maintenance. Here, we sought to identify the neural basis for such a perceptual memory, and whether any brain areas determined the likelihood that such signals would subsequently influence behavior.

Wellcome Trust Centre for Neuroimaging, University College London, UK magnetic resonance imaging to measure brain activity in a variable delay period of stimulus removal. Activity in the fusiform face area during the delay period following removal of rivalrous stimuli was greater following face than grating perception, whereas such a difference was absent during removal of nonrivalrous stimuli. Moreover, activity in areas of fronto-parietal regions during the delay period correlated with the degree to which individual participants tended to experience percept stabilization. Our findings suggest that percept-related activity in specialized extrastriate visual areas help to stabilize perception during perceptual conflict, and that high-level mechanisms may determine the influence of such signals on conscious perception.
We used binocular rivalry to investigate whether percept-specific brain activity occurred in a delay period after rivalrous stimuli had been physically removed, combining psychophysics and functional magnetic resonance imaging (fMRI) in two separate experiments. The rivaling stimuli were chosen such that one of the stimuli activated a specific region of functionally specialized visual cortex, where fMRI signals reflect perception during binocular rivalry (Tong, Nakayama, Vaughan, \& Kanwisher, 1998). This allowed us to use the signal in that region as a sensitive index of percept-specific activity. As a perceptual state tends to be maintained across periods of stimulus removal (Leopold et al., 2002), we hypothesized that percept-specific signals would be present not only during ongoing rivalry (Tong et al., 1998) but also during a delay period when the stimuli were absent. Moreover, top-down signals can alter the dynamics of rivalry and other forms of bistable perception (Meng \& Tong, 2004; Lack, 1978) and areas of nonvisual cortex are active during perceptual transitions (Sterzer, Russ, Preibisch, \& Kleinschmidt, 2002; Kleinschmidt, Büchel, Zeki, \& Frackowiak, 1998; Lumer, Friston, \& Rees, 1998). We thus hypothesized that percept maintenance (rather than just perceptual transitions) might also be associated with prefrontal and parietal activity. These two hypotheses are not mutually exclusive. Indeed, involvement of a distributed network of the frontal, parietal, and visual cortices in voluntary mnemonic 
processes such as working memory is well established (Petrides, 2005; Haxby, Petit, Ungerleider, \& Courtney, 2000), but would be unprecedented in the very different context of involuntary percept maintenance during bistable perception.

\section{METHODS}

\section{Behavioral Experiment}

Fourteen healthy volunteers aged 19-39 years (mean age $=27.6$ years, 9 men), with normal or corrected-tonormal vision, gave written informed consent to participate in the study which was approved by the local ethics committee. Visual stimuli were presented on a Dell UltraSharp 20-in. LCD monitor (refresh rate $60 \mathrm{~Hz}$ ) using MATLAB (Mathworks Inc.) and COGENT 2000 toolbox (www.vislab.ucl.ac.uk/cogent/index.html). Participants viewed stimuli from a distance of $60 \mathrm{~cm}$ through a mirror stereoscope in a semidark, quiet room. A chin-rest was used to minimize head movements. Sessions began with adjustment of the mirrors to allow binocular fusion of two small white dots each surrounded by white circles, presented on a gray background. The stimuli used in the experiment were a red-colored sinusoidal grating of 1.5 cycles/deg and a green-colored female face pre- sented on a gray background and subtending $2^{\circ}$ of visual angle (Figure 1A). The mean luminance of the stimuli was $42 \mathrm{Cd} / \mathrm{m}^{2}$. A white dot in the center and a white circle surrounding each stimulus facilitated fixation and binocular alignment. We chose a face stimulus for rivalry due to the strong selectivity of activity in regions of the fusiform gyrus for facial stimuli (Kanwisher, McDermott, \& Chun, 1997). This could therefore serve as a marker for any signals associated with perceptual fluctuations during ongoing rivalry (Andrews, Schluppeck, Homfray, Matthews, \& Blakemore, 2002; Tong et al., 1998; Kanwisher et al., 1997) or during a delay period when the stimuli were physically absent in the subsequent fMRI experiment.

The face stimulus was paired with a grating rather than another high-level stimulus because pilot experiments had shown that the occurrence of piecemeal rivalry was substantially reduced with the face/grating pair as compared to, for example, face/house pairs. For the same reason, the stimuli were rotating in opposite directions at a speed of 0.69 cycles/s (Haynes \& Rees, 2005) and differently colored.

There were two different experimental conditions: "rivalry" and "replay" (Figure 1A). During the rivalry condition, participants were simultaneously presented with the grating stimulus to one eye (right eye in 9 participants) and the face stimulus to the other eye.
Figure 1. Experimental design and behavioral data. (A) Behavioral experiment: During "rivalry," stimuli consisted of a green-colored face presented to one eye and a red-colored grating to the other eye. The sequence of 6-12 sec stimulus presentation ("stimulation"), 3-9 sec blank screen ("delay"), and again 3 sec stimulus ("probe") was repeated nine times while subjects used keypresses to report their percepts. During "replay," the subjects' perceptual sequence during stimulation in the rivalry condition was replayed by presenting monocular face and grating stimuli in alternation to the left and right eye. The probe stimulus was again rivalrous as in the rivalry condition. (B) Behavioral results $(n=14)$ : Percept maintenance, that is, the probability of the first percept upon onset of the probe

stimulus being the same as the last percept before the delay period, was significantly above chance (dashed line) for face and grating during rivalry but not during replay [** denotes $t(13)=9.6, p<.001$; * denotes $t(13)=2.4, p<.03$ ]. Error bars denote standard errors corrected for between-subject variability (Cousineau, 2007). (C) The experimental paradigm during the second fMRI experiment was similar to the psychophysical experiment. To maximize efficiency, visual stimulation alternated with blank delay periods without additional probe stimuli. Also, rivalry and replay conditions were presented in alternation within each experimental run rather than in separate runs. 
Participants continually indicated which perception was dominant (face or grating) using button presses. Rivalrous viewing (6-12 sec) was followed by a variable delay period of 3-9 sec with presentation of the white fixation dot and the white circle on a gray background. Upon subsequent presentation of the rivalrous face/grating stimulus ("probe") for $3 \mathrm{sec}$, participants were again required to indicate their perception by button press. The probe stimulus was followed by a 3-sec delay period. This sequence was repeated nine times per rivalry run. Stimulation and delay period durations were varied for similarity with the scanning experiment, where variable block durations were important for reasons of data analysis (see below). In a following replay run, participants were presented with nonrivalrous monocular face and grating stimuli shown in alternation to either eye, while the other eye was presented with the white fixation dot and the white circle on a gray background. The timing of these physical alternations mimicked the sequence of alternations reported in the preceding rivalry run. Face and grating stimulus presentations overlapped for $500 \mathrm{msec}$. As in the rivalry condition, blocks of replay stimulation were followed by a variable delay period where stimuli were physically removed, and then by the same rivalrous probe stimulus as in the rivalry condition. Again, one run comprised nine repetitions of the sequence stimulation-delay period-probe-delay period. Participants performed four to six pairs of rivalry/replay runs. This procedure allowed us to assess whether the percept at the onset of the rivalrous probe stimulus depended on whether the stimulation before the preceding delay period represented rivalrous stimulation or the control "replay" condition (note that the preceding perceptual sequences for each were identical). Importantly, the contrast of the stimuli was calibrated in each subject individually before the experiment in order to achieve equal percept probabilities at the onset of the rivaling stimuli.

\section{fMRI Experiment}

The same group of participants that had participated in the behavioral experiment subsequently underwent fMRI on a 3-T Siemens Allegra system using a standard head coil. We obtained blood-oxygenation-level-dependent (BOLD) echo-planar image volumes (38 contiguous transverse slices, voxel size $=3 \times 3 \times 3 \mathrm{~mm}$ ) with a TR (repetition time) of $2.47 \mathrm{sec}$. Stimuli were projected from an LCD projector (NEC LT158, refresh rate $60 \mathrm{~Hz}$ ) onto a screen at the head-end of the scanner that was viewed via a mirror attached to the head coil directly above the participants' eyes. The size of the screen was $10.5^{\circ} \times 8.4^{\circ}$ of visual angle. It was vertically split into two halves by a black cardboard divider that was placed between the screen and the mirror, thus separating images presented to the left and right eye. Perceptually, all participants reported that the two halves of the screen readily fused into one vertically oriented rectangle and that they had no problems maintaining binocular fusion throughout the experiment. The two binocular-rivalry stimuli were each projected onto the center of one halfscreen. The stimuli used were the same as in the preceding behavioral experiment (Figure 1C). The mean luminance of the stimuli was $55 \mathrm{Cd} / \mathrm{m}^{2}$.

The experimental paradigm during scanning was similar to the behavioral experiment, with the exception that, to maximize efficiency, visual stimulation simply alternated with variable delay periods, without separate probe trials (Figure 1C). Moreover, the rivalry and replay conditions were presented in alternation within each experimental run rather than in separate runs. Each rivalry and replay block comprised four alternations between stimulation and blank delay periods, and three pairs of rivalry/replay blocks were presented in each run (156 scan volumes). As in the behavioral experiment, the perceptual sequence as indicated by the participants' keypresses during each rivalry block was replayed with physical stimulus alternations in the following replay block. The entire experiment comprised six runs. Again, the contrast of the stimuli was calibrated prior to the experiment to achieve approximate equiprobability of either of the rivaling percepts at stimulus onset.

After the main experiment, a localizer scan (156 volumes) to functionally define the fusiform face area (FFA) according to standard criteria (Kanwisher et al., 1997) was performed. In brief, participants were presented with 12 sec blocks of either black-and-white faces or objects presented on a white screen $(500 \mathrm{msec}$ each with 500-msec interstimulus interval), interleaved with $8 \mathrm{sec}$ blocks of fixation baseline. In addition, we performed a T1-weighted structural scan (voxel size $1 \times 1 \times 1 \mathrm{~mm}$ ).

\section{Imaging Data Analysis}

Data were analyzed using statistical parametric mapping software (SPM2, www.fil.ion.ucl.ac.uk/spm). After discarding the first five image volumes from each run to allow for T1 equilibration effects, functional image volumes were realigned, unwarped (Andersson, Hutton, Ashburner, Turner, \& Friston, 2001), corrected for slice timing, spatially normalized into MNI neuroanatomical space (www.bic.mni.mcgill.ca/brainweb), and smoothed using a 10-mm full-width-at-half-maximum Gaussian kernel. We removed low-frequency fluctuations by a high-pass filter with a cutoff at 240 sec and used an autoregressive model of order one $(\operatorname{AR}(1)+$ white noise) to correct for temporal autocorrelation in the data. To minimize baseline differences across runs, the time series from each voxel was normalized to the mean signal across all scans of each run.

A mixed-effects analysis was adopted using a two-stage procedure. First, a participant-specific fixed-effects analysis was performed using the General Linear Model (GLM) implemented in SPM2 (Friston et al., 1995). This 
procedure fits the data with regressors that represent the experimental conditions to produce estimates at each voxel of the activation due to the experimental conditions. These parameter estimates represent the contribution of a particular regressor to the observed data. They are scaled to the average global signal across all scans and voxels and represent percent signal change with respect to the global brain signal. Regressors for face and grating percepts were modeled separately for rivalry and replay conditions based on the participants' keypresses. An additional regressor was created for mixed percepts lasting longer than $0.5 \mathrm{sec}$ (which occurred rarely; average duration of mixed percepts across participants $0.46 \mathrm{sec} \pm 0.26$ SEM). Separate regressors were explicitly modeled for delay periods following face and grating perception during both rivalry and replay and an additional regressor in the rare event of a mixed percept directly preceding the delay period. The evoked responses were modeled as boxcar functions convolved with a canonical hemodynamic response function as implemented in SPM2. For each participant, $t$ maps of the eight effects of interest (face and grating perception during visual stimulation in rivalry and replay; and delay period following face and grating perception in rivalry and replay) were computed. Either individual parameter estimates from the FFA or whole-brain $t$ maps were then submitted to second-level random-effects analyses, where effects were assessed using a repeated measures analysis of variance (ANOVA).

\section{Brain Activity Associated with Rivalry-specific Percept Maintenance}

First, we determined whether there was a correlate of rivalry-specific percept maintenance in percept-related extrastriate visual areas. In the absence of evidence for a brain region preferentially processing gratings rather than faces, this analysis focused on the FFA, which was defined in each participant individually using standard criteria (Kanwisher et al., 1997), as the mid-fusiform activation cluster showing the strongest activations in the contrast (faces - objects) in the separate functional localizer scan. It was located in the right fusiform gyrus in 11 participants and in the left in 3 participants. Parameter estimates for the eight conditions of interest in the main experiment were extracted from the peak voxel (which, due to spatial smoothing, represents a weighted average of nearby voxels) in each participant's functionally localized FFA and subjected to statistical inference at the group level, using paired two-tailed $t$ tests and repeated measures two- and three-way ANOVA, where appropriate.

Face- and rivalry-specific effects were also assessed in a conventional random-effects analysis using the contrast $\left(\right.$ face $_{\text {delay }}-$ grating delay $_{\text {rivalry }}-\left(\text { face }_{\text {delay }}-\text { grating delay }\right)_{\text {replay }}$. To test for effects in regions responding more strongly to face than grating perception, this analysis was lim- ited to voxels showing a significant effect for the contrast $\left(\text { face }_{\text {rivalry }}+\text { face }_{\text {replay }}\right)_{\text {stimulation }}-$ (grating $_{\text {rivalry }}+$ grating $\left._{\text {replay }}\right)_{\text {stimulation, }}$, thresholded at $p<.05$, uncorrected. The mid-fusiform activation clusters resulting from this inclusive masking procedure (number of voxels $k=$ 170 and $k=67$ in right and left fusiform gyrus, respectively) were used for small-volume correction. Effects were reported if they were significant at $p<.05$, using a family-wise error (FWE) correction for multiple comparisons. To rule out the possibility that any such interaction effects were nonspecific, that is, effects seen throughout the visual cortex and not limited to faceresponsive regions, we performed a similar analysis in regions that were nonspecifically activated by both face and grating stimuli. Voxels generally responsive to visual stimulation were identified by contrasting all stimulation periods with all delay periods ([face rivalry + face $_{\text {replay }}+$ grating $_{\text {rivalry }}+$ grating $\left._{\text {replay }}\right]_{\text {stimulation }}-\left[\right.$ face $_{\text {rivalry }}+$ face $_{\text {replay }}+$ grating $_{\text {rivalry }}+$ grating $\left.\left._{\text {replay }}\right]_{\text {delay }}\right)$. We then tested for interaction effects as above ([face delay $_{\text {- grat- }}$ ing $\left.\left._{\text {delay }}\right]_{\text {rivalry }}-\left[\text { face }_{\text {delay }}-\text { grating delay }\right]_{\text {replay }}\right)$ using smallvolume correction for spheres of $10 \mathrm{~mm}$ diameter $(k=$ 73 voxels) centered on the coordinates of the peak voxels activated by visual stimulation versus delay bilaterally in the occipital cortex. Again, interaction effects were considered significant at $p<.05$, using FWE correction in these regions of interest.

We also investigated whether percept maintenance might be associated with activity in frontal and parietal regions, especially in those that are known to be involved in working memory, using the contrast (face $_{\text {delay }}+$ grating delay $)_{\text {rivalry }}-\left(\text { face }_{\text {delay }}+\text { grating }_{\text {delay }}\right)_{\text {replay. }}$ We also tested for activations specific to face maintenance during rivalry, again using the interaction term (face $_{\text {delay }}-$ grating delay $_{\text {rivalry }}-\left(\right.$ face $_{\text {delay }}-$ grating delay $_{\text {replay. }}$ Based on the previous literature (Sala, Rama, \& Courtney, 2003; Haxby et al., 2000; Courtney, Ungerleider, Keil, \& Haxby, 1996, 1997), frontal regions that were expected to show such activations included the inferior frontal gyrus/anterior insula, the posterior mid-frontal cortex, and the anterior mid-frontal cortex. Parietal activations during face maintenance were hypothesized to be located in the region of the intraparietal sulcus. Effects were considered significant at $p<.05$, using an FWE correction for multiple comparisons across the whole brain, or at $p<.001$, uncorrected, if predicted by our a priori hypothesis.

\section{Brain Activity Predicting Percept Maintenance across Participants}

Next, we determined whether each individual's behavioral propensity to maintain a percept correlated with activations in any of our hypothesized brain regions. Each participant's rivalry-specific tendency to maintain a percept across the variable delay period was defined as the percentage of maintained percepts (face + grating) 
across the delay period during rivalry minus the percentage of maintained percepts during replay in the psychophysical experiment. Importantly, we did not use the percentages of maintained face-percepts even when testing for correlations with face-specific brain activations. The percept-independent maintenance effect was considered a better surrogate marker for individual performance because it was not confounded by slight imbalances in percept-onset probabilities in individual participants (resulting, e.g., from slow drifts in contrast sensitivity). Such slight imbalances canceled out across the whole group, but might have led to a misrepresentation of an individual's tendency for percept maintenance. Correlation effects were assessed for the same contrasts that were used to analyze group effects, that is, $\left(\right.$ face $_{\text {delay }}+$ grating delay $_{\text {rivalry }}-\left(\right.$ face $_{\text {delay }}+$ grating $\left._{\text {delay }}\right)_{\text {replay }}$ and $\left(\text { face }_{\text {delay }}-\text { grating }_{\text {delay }}\right)_{\text {rivalry }}-$ $\left(\text { face }_{\text {delay }}-\text { grating }_{\text {delay }}\right)_{\text {replay }}$, applying the same criteria for statistical significance. In addition to voxelwise whole-brain analyses, we also tested for correlations between percept maintenance and face-specific effects in the FFA as localized in each participant individually.

\section{RESULTS}

\section{Experiment 1: Perceptual Memory is Specific to Bistable Perception}

In this purely behavioral experiment, subjects were either presented with monocular images of a face and grating that resulted in binocular rivalry ("rivalry" condition); or with the identical face and grating stimuli in physical alternation, replaying the perceptual time course as indicated by button presses during the preceding rivalry condition ("replay"). After a short period of viewing, the stimuli were removed and a variable delay period of several seconds ensued, followed by the presentation of a rivalrous "probe" stimulus. The stimulus contrasts were adjusted to achieve equal overall probabilities of face and grating percepts at the onset of rivalrous stimuli $[0.49 \pm 0.04$ SEM and $0.44 \pm 0.05$ SEM, respectively; $t(13)=0.8, p<.44$, paired two-tailed $t$ test]. The proportion of mixed (piecemeal) percepts at stimulus onset was low $(0.07 \pm 0.03$ SEM) .

Perception of the probe stimulus was strongly and significantly influenced by the last reported percept of the preceding rivalrous stimulus, replicating previous findings (Leopold et al., 2002). In contrast, the preceding replay stimulus had no detectable influence on perception of the subsequent rivalrous probe stimulus (Figure 1B). The overall percentage of maintained percepts was $70.9 \pm 3.4 \%$ SEM (chance level $=50 \%$ ) in rivalry and $48.5 \pm 2.5 \% S E M$ in replay $[t(13)=7.2$, $p<.001]$. There was a trend toward a stronger rivalryspecific maintenance effect for face compared to grating stimuli $[F(1,13)=4.4, p=.057]$. Involuntary percept maintenance across a variable delay period of stimulus removal was therefore specific to binocular rivalry and not seen after temporally identical sequences of physical stimulus alternation.

\section{Experiment 2: A Correlate of Percept Maintenance in the Ventral Visual Cortex}

We next asked whether this involuntary percept maintenance across delay periods where the stimuli were absent was accompanied by any brain activity during these delay periods that encoded what had previously been perceived at the moment of stimulus disappearance. The experimental paradigm during scanning was similar to the psychophysical experiment (Figure 1C). Stimulus contrasts were again adjusted prior to the experiment to match the probabilities of face and grating percepts at the onset of the rivaling stimuli $[0.50 \pm$ 0.04 SEM and $0.45 \pm 0.05$ SEM, respectively; $t(13)=$ $0.55, p<.59$, paired two-tailed $t$ test], again with a low proportion of mixed percepts at onset $(0.05 \pm 0.03$ $S E M)$. Again, overall percept maintenance across variable delay periods following rivalry was significantly above chance $[59.1 \pm 2.2 \%, t(13)=4.1, p<.001]$, but could not be directly compared to the replay condition because no probe trials were used during scanning (see Methods and Figure 1).

We first examined brain activity in each participant's independently identified FFA. During rivalry, we observed robustly and significantly elevated activity when participants reported conscious perception of a face, compared to periods when they reported grating perception (Figure 2A), replicating previous findings (Tong et al., 1998). Overall, there was a trend toward a significant main effect for greater FFA activity in rivalry compared to replay $[F(1,13)=4.6, p=.053]$, but the activity difference between face and grating perception was equal during both conditions $[F(1,13)=0.02$, $p=.90$ for a two-way interaction]. Thus rivalry and replay were equivalent in their percept-related effects on the FFA during perceptual fluctuations (Tong et al., 1998).

Critically, our use of intermittent variable delay periods now allowed us to also characterize FFA activity in the absence of visual stimulation, and to test whether such activity was specific for the delay periods following binocular rivalry (where percept maintenance occurred behaviorally) as opposed to those following replay stimuli (where no percept maintenance had been observed). In contrast to the findings during visual stimulation, where FFA activity differences between face and grating perception had been similar in rivalry and replay, differential FFA activation during the variable delay period was only observed following rivalrous stimuli, but not following replay. Specifically, FFA activation in the absence of visual stimulation following rivalrous perception was significantly higher when the last stimulus in awareness before disappearance was a face, rather than a grating [Figure 2B: $t(1,13)=3.3, p<.006$ ]. This effect was specific to rivalry, as FFA activity during blank delay 


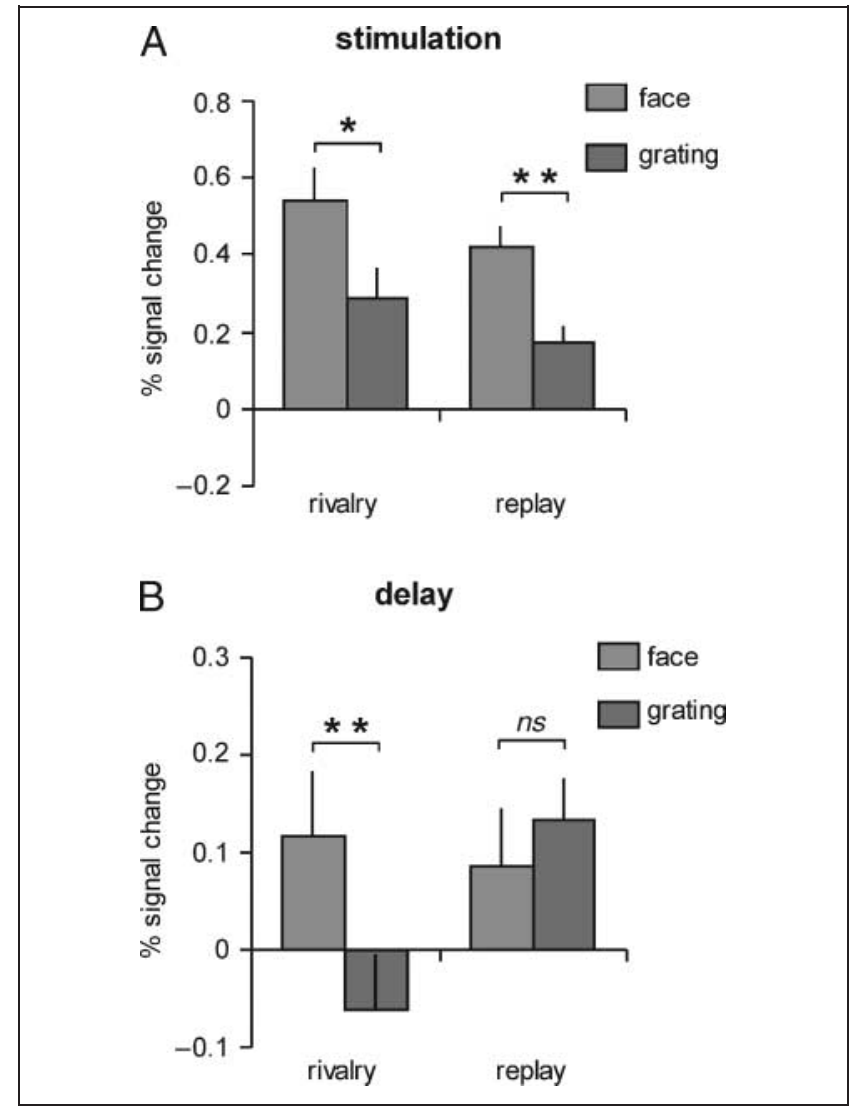

Figure 2. FFA activity during visual stimulation and delay periods. Average parameter estimates of brain activity (\% of global brain signal, for details of parameter estimation, see Methods) extracted from the FFA of each of 14 participants, as determined in a separate localizer scan. $* p<.05 ; * *<.01 ; n s=$ not significant. (A) During visual stimulation, FFA activity was greater during face (green) than during grating (red) perception in both "rivalry" and "replay" $\left[t(13)=2.6, p<.02\right.$ for $\left(\text { face }_{\text {stimulation }}-\text { grating }_{\text {stimulation }}\right)_{\text {rivalry }}$; $\left(t(13)=3.5, p<.004\right.$ for $\left.\left(\text { face }_{\text {stimulation }}-\text { grating }_{\text {stimulation }}\right)_{\text {replay }}\right]$. (B) During delay periods in the rivalry condition, FFA activity following face perception was greater than following grating perception $\left[t(13)=3.3, p<.006\right.$ for $\left.\left(\text { face }_{\text {delay }}-\text { grating }_{\text {delay }}\right)_{\text {rivalry }}\right]$. Such a difference was absent during replay $[t(13)=-1.1, p<.29$ for $\left(\text { face }_{\text {delay }}-\text { grating }_{\text {delay }}\right)_{\text {replay }} ;(1,13)=11.5, p<.005$ for a two-way interaction]. Error bars denote standard errors corrected for between-subject variability (Cousineau, 2007).

periods following physical alternations of the stimuli did not show such a difference [Figure 2B: $t(1,13)=-1.1$, $p<.29$ ]. Moreover, the interaction between condition (rivalry or replay) and which stimulus was present in awareness immediately prior to the delay period (face or grating) was significant $[F(1,13)=11.5, p>.005]$, and this interaction was specific for the variable delay period as opposed to periods of physical stimulation $[F(1,13)=5.55, p<.035$ for a three-way interaction $]$.

The rivalry-specific activity difference during variable delay periods is illustrated by both the parameter estimates (Figure 2B) and the plots of the averaged eventrelated signal time courses (Figure 3). Note, however, that the duration of the delay period was varied (3-9 sec) to achieve a high degree of orthogonality between regressors representing delay period and visual stimulation conditions, thereby maximizing design efficiency for analysis using the GLM (Friston et al., 1995) (see Methods). With the caveat that the interpretability of the time course plots is therefore limited, they nevertheless also suggest a clear difference in overall activity comparing delay periods following face perception with those following grating perception during rivalry up to 3 scan volumes $(=7.4 \mathrm{sec})$ after the offset of visual stimulation. Critically, such a difference is not present in the replay condition and, therefore, the differences observed following rivalry cannot reflect the effects of physical stimulation carrying over into the delay period. Importantly, our statistical analyses were based on parameter estimates (Figure 2) derived from the GLM, where differences in duration are taken into account, and so the activity differences we report reflect the correlation of rivalry-specific stabilization with the amplitude of delayperiod activity, irrespective of the duration of a particular delay period. Moreover, all conditions were explicitly modeled as separate regressors in one model, such that signal changes due to preceding and subsequent visual

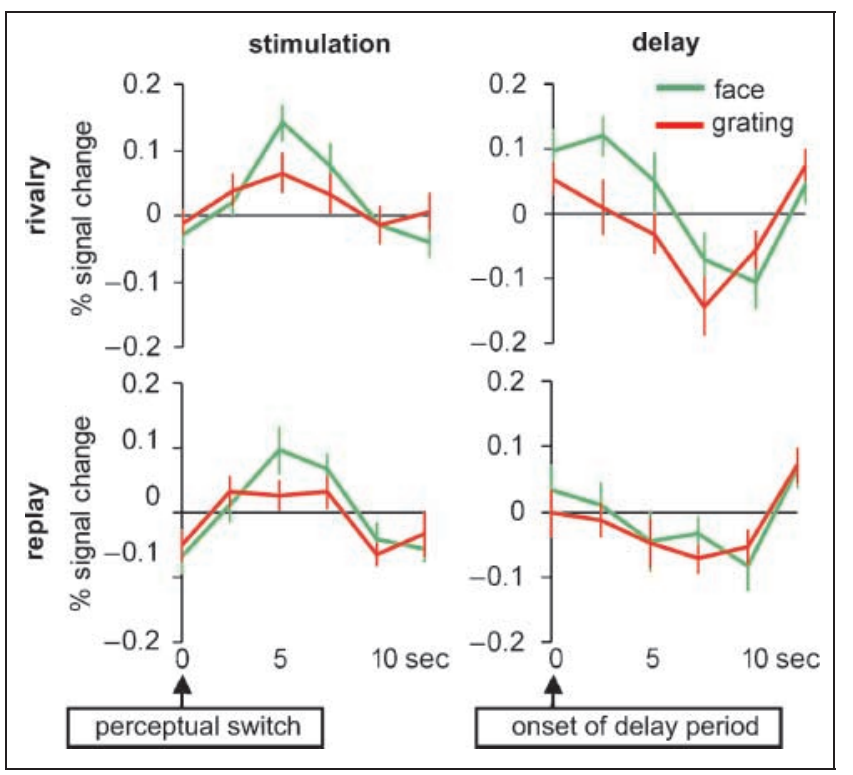

Figure 3. Event-related signal time courses. Averaged fMRI signal time courses from each individual's FFA time-locked to perceptual switches during visual stimulation and to onsets of delay periods, respectively. The fMRI time series of each voxel were normalized to the mean across all scans of each run (see Methods). The averaged time courses shown, therefore, represent percent signal change relative to the mean signal across all conditions. Because perceptual switches as well as delay periods were randomly jittered with respect to scan onsets, the time-course data were binned into TRs ( TR $=$ repetition time for fMRI measurements, $2.47 \mathrm{sec}$ ). Activation differences between face (green) and grating (red) perception in rivalry and replay were similar during visual stimulation, whereas during delay periods following face and grating perception such a difference was only present in rivalry. Error bars denote standard errors corrected for between-subject variability (Cousineau, 2007). 
stimulation were also accounted for, and thus, did not confound the estimation of activity differences during the blank delay periods (Friston et al., 1995). The consistency of parameter-estimate-related and time-course data lends strength to this external validation that the GLM approach was effective at distinguishing stimulusdriven activity from delay-period-related activity.

The face- and rivalry-specific effect during delay periods was identified both when analyzing data from each participant's FFA as defined in a separate functional localizer experiment (Figure 2), and for a voxelwise analysis [Figure 4; right: $x y z=40-50-15, t(13)=2.64$, $p<.035$, small-volume correction; left: $x$ y $z=-44-56$ $-14, t(13)=2.55, p<.026$, small-volume correction]. Importantly, such an effect was not found in regions activated by both face and grating stimuli. Peak activations in response to visual stimulation independently of stimulus type were located bilaterally in the extrastriate visual cortex, slightly lateral to the occipital pole (right: $x y z=36-84-6$; left: $x y z=-38-84-4)$. When performing a random-effects analysis similar to the one in the FFA in regions of interest centered on these peak activations, we failed to find any significant face- and rivalry-specific effect during delay periods [right: $t(13)=$ $1.71, p>.1$, small-volume correction; left: $t(13)=1.51$, $p>.1$, small-volume correction], demonstrating that the

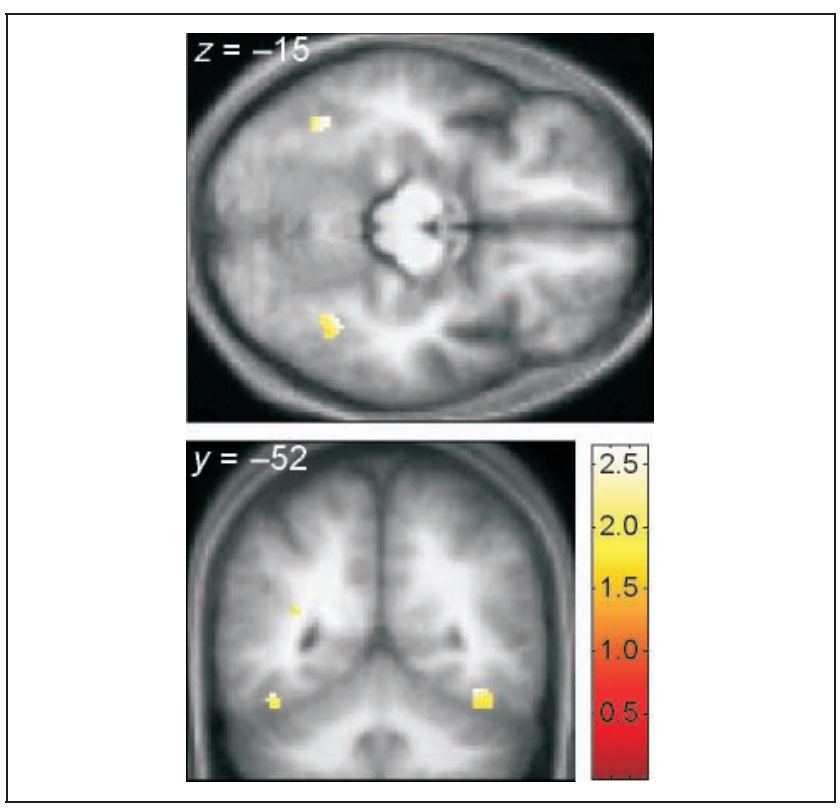

Figure 4. Voxelwise analysis of face-specific activity during delay periods. A group statistical parametric map showing bilateral fusiform activation foci where activity was significantly greater during blank delay periods following face (vs. grating) perception for rivalry (vs. replay), that is, the statistical contrast (face delay $_{-}$grating delay) rivalry $\left(\text { face }_{\text {delay }}-\text { grating delay }\right)_{\text {replay. }}$ Group activations are rendered onto transversal and coronal sections of the mean structural scan across participants and thresholded at $p<.05$, using inclusive masking with the main effect for face versus grating perception at $p<.05$ (see Methods). The significance levels ( $t$ scores) are color-coded as indicated in the color bar. reported interaction effect was indeed specific to faceresponsive regions.

\section{Activity in Frontal and Parietal Regions Correlates with Individual Percept Maintenance}

Next, we further investigated our hypothesis that the amplitude of activity in either visual or higher-order areas during the delay period might correlate with the probability of percept maintenance. As in previous studies (Leopold et al., 2002), the tendency of participants to maintain perception across intermittent rivalrous stimulation varied substantially ( $\sim 5$ to $45 \%$ difference between rivalry and replay in average percept maintenance). We therefore exploited this natural variability in behavior in order to determine whether these interindividual differences might be associated with the amplitude of activity during blank delay periods in brain regions potentially involved in percept maintenance. We found no significant correlations between individual rivalry-specific percept maintenance and rivalry-specific brain activity across percept types. In contrast, highly significant face-specific effects, that is, correlations between individual percept maintenance and rivalry-related activity during the delay periods following face as opposed to grating percepts, were identified in regions of the frontal and parietal cortex. Most prominently, there was an almost-perfect correlation in the right anterior middle frontal gyrus [Figure 5; $x y z=324022, r=.93, t(13)=8.82, p<.036$, corrected for multiple comparisons across the whole brain]. At a more lenient statistical threshold, correlations between brain activity and individual behavior were also observed

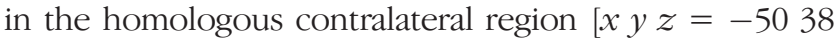
$18, r=.75, t(13)=3.93, p<.001$, uncorrected $]$ and in the anterior portion of the intraparietal sulcus bilaterally [x y $z=30-5438, r=.78, t(13)=4.26, p<.001$, uncorrected; $x$ y $z=-42-3850, r=.77, t(13)=4.23$, $p<.001$, uncorrected]. Thus, the amplitude of activity in these areas during the blank delay period following rivalrous face perception strongly correlated with the probability that a participant would subsequently perceive a face when the rivalrous stimuli reappeared, as assessed behaviorally. Comparing rivalry and replay across the whole group of participants, activity differences in these brain regions did not reach statistical significance ( $p<.001$, uncorrected), neither generally nor specifically in the delay period following face perception. In contrast, activity in the FFA, where we had found face-specific activity across the whole group, showed no significant correlation between behavioral percept maintenance and the face-specific effect of rivalry during delay periods $(r=.28$, $p<.34)$

\section{DISCUSSION}

We investigated percept stabilization in binocular rivalry using an established behavioral paradigm (Leopold et al., 
Figure 5. Neural correlates of successful percept maintenance following rivalry in the prefrontal and parietal cortex. Displayed are activated foci that showed a positive correlation between rivalry-specific percept survival (\% maintenance rivalry $_{-}$ $\%$ maintenance replay $_{\text {) }}$ and rivalry-specific activity differences between delay periods following face perception and those following grating perception face $_{\text {delay }}-$ grating delay $)_{\text {rivalry }}-\left(\right.$ face $_{\text {delay }}-$ grating $\left._{\text {delay }}\right)_{\text {replay. }}$ The activation maps are rendered onto sections of the mean structural scan across participants and for display purposes thresholded at $p<.005$, uncorrected. The significance levels ( $t$ scores) are color-coded as indicated in the color bar. The regression plot shows the correlation between rivalry-specific percept maintenance and the difference in brain activity for the statistical contrast (face $e_{\text {delay }}-$ grating $\left._{\text {delay }}\right)_{\text {rivalry }}-\left(\right.$ face $_{\text {delay }}-$ grating delay) $_{\text {replay }}$ at the peak voxel in right anterior middle frontal gyrus (white circles on the activation maps). Each point represents data from a single participant. Note that the $p$ value given is family-wise error (FWE)-corrected for multiple comparisons across the whole brain.
2002), where a perceptual state tends to be maintained across periods of temporary stimulus removal. We showed behaviorally that this percept-stabilizing effect was specific to binocular rivalry as compared to a situation without perceptual conflict. Using fMRI, we demonstrated that percept maintenance was associated with percept-specific signals in the functionally specialized visual cortex and that, across participants, the tendency to experience a maintained percept after a delay correlated with activity in prefrontal and parietal regions.

Our behavioral finding that percept maintenance across blank delay periods was specific to binocular rivalry suggests that percept maintenance depended on the persistence of an internally generated perceptual state during rivalry, rather than being a nonspecific effect of perceptual priming or "inertia" (as previously demonstrated for ambiguous patterns preceded by biasing stimuli; Pinkus \& Pantle, 1997; Long, Toppino, \& Mondin, 1992). Consistent with this, rivalrous and nonrivalrous (unambiguous) stimuli briefly presented during a delay period following rivalry differentially affect the maintenance effect (Pearson \& Clifford, 2005). Taken together, these behavioral findings indicate that binocular rivalry recruits mechanisms for percept stabilization that are not engaged when visual competition is minimized (as with our "replay" condition). That is, once perceptual conflict has been resolved in favor of one percept, such mechanisms seem to promote the maintenance of the current resolution of the conflict even in the absence of visual stimulation. This suggests that percept maintenance in binocular rivalry-and presumably also in other forms of bistable perceptioninvolves involuntary "mnemonic" processes dedicated to the resolution of visual competition or ambiguity.

We predicted that, if percept-specific mechanisms were involved in percept maintenance, we should find a percept-specific signal during delay periods after rivalry even though no stimuli were physically present. In accordance with this prediction, we observed such a "mnemonic" signal in the FFA, that is, greater activity during delay periods following face perception than during those following grating perception, whereas such a difference was absent in the replay condition. This percept-specific "mnemonic" signal was specific to the FFA, as it was not seen in regions of the occipital cortex that responded to both face and grating stimuli. Of note, the difference in FFA activity between face and grating perception during visual stimulation was of equal magnitude in the rivalry and replay conditions (Figure 2A), replicating earlier findings (Tong et al., 1998). The signal difference observed during the delay periods following rivalrous stimulation (Figure 2B), therefore, cannot result from a carryover, or slow resolution, of activity differences in the immediately preceding stimulation period, as such nonspecific effects were not identified in delay periods following replay. Differences in attention or alertness between rivalry and replay conditions also cannot account for the observed effect, as there was 
no overall difference in FFA activity comparing rivalry versus replay during the variable delay periods. Nonspecific mechanisms, such as imagery or attempts to actively maintain a percept, are also unlikely to have caused the signal difference in the FFA, as participants were only required to passively view the stimuli and report their percepts.

The association of FFA activity with percept maintenance does not seem to be due to increased activity during delay periods following face perception (see Figure 2B). Rather, when comparing the effect sizes during delay periods in rivalry and replay, FFA activity seems decreased following grating perception in rivalry (but not in replay). The negative parameter estimate per se cannot be interpreted as suppression of neural activity because the parameter estimates do not represent absolute effects but are scaled relative to the global signal (see Methods). However, the relative decrease compared to all other conditions, nevertheless, suggests relative suppression. This could indicate that the suppression of the neural representation of the nondominant stimulus that is believed to occur during ongoing rivalry (Blake \& Logothetis, 2002) might also persist during stimulus interruptions, thereby generating percept maintenance across these interruptions. Computational models of binocular rivalry assume that dominance and suppression can be generated by competitive interactions between dynamic neuronal assemblies at various stages of the visual processing hierarchy (Wilson, 2003; Dayan, 1998). Empirically, rivalry can be partially or completely resolved between monocular cells early in visual processing (Haynes, Deichmann, \& Rees, 2005; Wunderlich, Schneider, \& Kastner, 2005; Tong \& Engel, 2001), but can also reflect interstimulus rather than interocular competition (Kovacs, Papathomas, Yang, \& Feher, 1996; Logothetis, Leopold, \& Sheinberg, 1996), and thus, probably involves multiple processes implemented at different neural levels (Wilson, 2003; Blake \& Logothetis, 2002). In intermittent rivalry, eye-of-origin information is an important determinant of percept maintenance across stimulus interruptions (Chen \& He, 2004; Pearson \& Clifford, 2004). However, perceptually relevant factors, such as the color or orientation of competing grating stimuli, also have a significant influence on percept maintenance (Pearson \& Clifford, 2004). Thus, the mnemonic process during interruption of rivalrous stimuli is presumably also implemented at different stages of the visual processing hierarchy, similar to ongoing rivalry.

In addition to eye- and percept-related mechanisms, whether a percept is maintained across a delay period is also determined by local adaptation (Chen \& He, 2004; Blake, Sobel, \& Gilroy, 2003), and presumably by feature- or object-based attention (Chong \& Blake, 2005; Meng \& Tong, 2004; Mitchell, Stoner, \& Reynolds, 2004) and other less well-explored factors such as slow drifts in contrast sensitivity and fluctuations in arousal. Importantly, we found strong evidence that the individual tendency to maintain a percept during rivalry was associated with activity in prefrontal and parietal regions. Very similar loci have been previously implicated in the voluntary engagement of working memory for faces (Sala et al., 2003; Haxby et al., 2000; Courtney et al., 1996, 1997). In contrast to working memory tasks, percept maintenance in intermittent bistable perception occurs involuntarily without any conscious effort. We did not explicitly manipulate working memory in this study and cannot, therefore, make any definitive statement about the possible involvement of working memory-like processes in bistable perception. Nevertheless, our findings suggest that percept maintenance is influenced by higher-order (as opposed to only sensory) mechanisms and that these mechanisms share a common anatomical substrate with working memory processes.

Activity in frontal and parietal regions did not correlate with rivalrous percept maintenance overall (i.e., for delay periods following both face and grating perception), but specifically for delay periods following face perception as compared to grating perception. One possible interpretation is that higher-order mechanisms that govern the maintenance of face, as opposed to grating, percepts might differ or even interact in a competitive fashion. This could be underpinned by categorical stimulus differences. For example, a rotating grating will be much less likely to be processed as a coherent object than a face stimulus. Accordingly, face-specific activations in prefrontal regions have been observed in response to face stimuli (Ishai, Schmidt, \& Boesiger, 2005; Ishai, Pessoa, Bikle, \& Ungerleider, 2004; Scalaidhe, Wilson, \& Goldman-Rakic, 1999) and during working memory for faces (Sala et al., 2003; Haxby et al., 2000; Scalaidhe et al., 1999; Courtney et al., 1996, 1997). However, whether frontal and parietal activations observed during working memory are specific for the type of stimulus remains controversial (Haxby et al., 2000; Owen, 2000). It therefore remains an intriguing topic for future research whether our observations of facespecific mnemonic activations following rivalry might, indeed, reflect involvement of a more general neural mechanism.

In contrast to activity in prefrontal and parietal regions, the amplitude of FFA activity during delay periods did not significantly correlate with the behavioral probability of percept maintenance. This may seem surprising, as one might expect higher-order regions to affect percept maintenance by top-down control of visual areas representing the conflicting stimuli. However, it is conceivable that such putative top-down influences may not be exerted by boosting the representation of the dominant stimulus, but rather by enhancing suppression of the nondominant one. Although such an interpretation must remain speculative, our findings may, nevertheless, indicate that percept-related activity differences in visual areas might be necessary but, in themselves, not sufficient for percept maintenance in binocular rivalry. 
Taken together, our findings suggest a model whereby involuntary percept stabilization during binocular rivalry is associated with percept-specific signals in the specialized extrastriate visual cortex; but the likelihood that such signals will subsequently determine conscious perception depends on activity in the parietal and prefrontal cortex. However, our observational data cannot confirm a causal role for such regions without further experimentation. Nevertheless, they add a new aspect to the ongoing debate about the roles of higher-level regions, especially in the prefrontal cortex, versus lowerlevel sensory processing areas in selecting sensory information for conscious awareness. It has been proposed that activity in sensory processing areas is necessary but, in itself, not sufficient to produce a conscious percept, and that selection of sensory information for consciousness requires top-down amplification from higher-order brain regions (Maia \& Cleeremans, 2005; Crick \& Koch, 2003; Rees, Kreiman, \& Koch, 2002; Dehaene \& Naccache, 2001). Here, we focused on persistent brain activity in the absence of visual input, and thus, also in the absence of awareness of particular percepts. We assessed the influence of such brain activity not on the selection of a conscious percept but on the maintenance of a perceptual "set" across periods of stimulus absence. Consistent with current models of conscious visual awareness, we propose that activity in sensory areas is necessary but not sufficient to stabilize perception across periods of stimulus absence and that frontal and parietal regions act to determine the influence of such activity on percept stabilization. Our findings may thus extend the role of interactions between higher-level and sensory brain regions to the stabilization of the current contents of awareness. Overall, we therefore conclude that an active and automatic mnemonic process may serve to stabilize conscious perception whenever our brains are confronted with the task of constructing a unified percept from ambiguous or conflicting information.

\section{Acknowledgments}

This work was funded by the Wellcome Trust (G. R.) and the Deutsche Forschungsgemeinschaft (P. S.). We thank Jon Driver, Chris Frith, and John-Dylan Haynes for helpful comments on an earlier version of the manuscript.

Reprint requests should be sent to Philipp Sterzer, Department of Psychiatry, Charité University Hospital/St Hedwig Hospital, Große Hamburger Straße 5-11, D-10115 Berlin, Germany, e-mail: philipp.sterzer@charite.de.

\section{REFERENCES}

Andersson, J. L., Hutton, C., Ashburner, J., Turner, R., \& Friston, K. (2001). Modeling geometric deformations in EPI time series. Neuroimage, 13, 903-919.

Andrews, T. J., Schluppeck, D., Homfray, D., Matthews, P., \& Blakemore, C. (2002). Activity in the fusiform gyrus predicts conscious perception of Rubin's vase-face illusion.

Neuroimage, 17, 890-901.
Blake, R., \& Logothetis, N. K. (2002). Visual competition. Nature Reviews Neuroscience, 3, 13-21.

Blake, R., Sobel, K. V., \& Gilroy, L. A. (2003). Visual motion retards alternations between conflicting perceptual interpretations. Neuron, 39, 869-878.

Chen, X., \& He, S. (2004). Local factors determine the stabilization of monocular ambiguous and binocular rivalry stimuli. Current Biology, 14, 1013-1017.

Chong, S. C., \& Blake, R. (2005). Exogenous attention and endogenous attention influence initial dominance in binocular rivalry. Vision Research, 46, 1794-1803.

Courtney, S. M., Ungerleider, L. G., Keil, K., \& Haxby, J. V. (1996). Object and spatial visual working memory activate separate neural systems in human cortex. Cerebral Cortex, 6, 39-49.

Courtney, S. M., Ungerleider, L. G., Keil, K., \& Haxby, J. V. (1997). Transient and sustained activity in a distributed neural system for human working memory. Nature, 386, 608-611.

Cousineau, D. (2007). Confidence intervals in within-subject designs: A simpler solution to Loftus and Masson's method. Tutorials in Quantitative Methods for Psychology, 1, 42-45.

Crick, F., \& Koch, C. (2003). A framework for consciousness. Nature Neuroscience, 6, 119-126.

Dayan, P. (1998). A hierarchical model of binocular rivalry. Neural Computing, 10, 1119-1135.

Dehaene, S., \& Naccache, L. (2001). Towards a cognitive neuroscience of consciousness: Basic evidence and a workspace framework. Cognition, 79, 1-37.

Friston, K. J., Holmes, A. P., Worsley, K. P., Poline, J. B., Frith, C. D., \& Frackowiak, R. S. (1995). Statistical parametric maps in functional imaging: A general linear approach. Human Brain Mapping, 2, 189-210.

Haxby, J. V., Petit, L., Ungerleider, L. G., \& Courtney, S. M. (2000). Distinguishing the functional roles of multiple regions in distributed neural systems for visual working memory. Neuroimage, 11, 145-156.

Haynes, J. D., Deichmann, R., \& Rees, G. (2005). Eye-specific effects of binocular rivalry in the human lateral geniculate nucleus. Nature, 438, 496-499.

Haynes, J. D., \& Rees, G. (2005). Predicting the stream of consciousness from activity in human visual cortex. Current Biology, 15, 1301-1307.

Ishai, A., Pessoa, L., Bikle, P. C., \& Ungerleider, L. G. (2004). Repetition suppression of faces is modulated by emotion. Proceedings of the National Academy of Sciences, U.S.A., 101, 9827-9832.

Ishai, A., Schmidt, C. F., \& Boesiger, P. (2005). Face perception is mediated by a distributed cortical network. Brain Research Bulletin, 67, 87-93.

Kanwisher, N., McDermott, J., \& Chun, M. M. (1997). The fusiform face area: A module in human extrastriate cortex specialized for face perception. Journal of Neuroscience, 17, 4302-4311.

Kleinschmidt, A., Büchel, C., Zeki, S., \& Frackowiak, R. S. J. (1998). Human brain activity during spontaneously reversing perception of ambiguous figures. Proceedings of the Royal Society of London, Series B, 265, 2427-2433.

Kovacs, I., Papathomas, T. V., Yang, M., \& Feher, A. (1996). When the brain changes its mind: Interocular grouping during binocular rivalry. Proceedings of the National Academy of Sciences, U.S.A., 93, 15508-15511.

Lack, L. (1978). Selective attention and the control of binocular rivalry. Hague: Mouton.

Leopold, D. A., Wilke, M., Maier, A., \& Logothetis, N. K. (2002). Stable perception of visually ambiguous patterns. Nature Neuroscience, 5, 605-609. 
Logothetis, N. K., Leopold, D. A., \& Sheinberg, D. L. (1996). What is rivalling during binocular rivalry? Nature, 380, 621-624.

Long, G. M., Toppino, T. C., \& Mondin, G. W. (1992). Prime time: Fatigue and set effects in the perception of reversible figures. Perception \& Psychophysics, 52, 609-616.

Lumer, E. D., Friston, K. J., \& Rees, G. (1998). Neural correlates of perceptual rivalry in the human brain. Science, 280, 1930-1933.

Maia, T. V., \& Cleeremans, A. (2005). Consciousness: Converging insights from connectionist modeling and neuroscience. Trends in Cognitive Sciences, 9, 397-404.

Meng, M., \& Tong, F. (2004). Can attention selectively bias bistable perception? Differences between binocular rivalry and ambiguous figures. Journal of Vision, 4, 539-551.

Mitchell, J. F., Stoner, G. R., \& Reynolds, J. H. (2004). Object-based attention determines dominance in binocular rivalry. Nature, 429, 410-413.

Owen, A. M. (2000). The role of the lateral frontal cortex in mnemonic processing: The contribution of functional neuroimaging. Experimental Brain Research, 133, 33-43.

Pearson, J., \& Clifford, C. G. (2004). Determinants of visual awareness following interruptions during rivalry. Journal of Vision, 4, 196-202.

Pearson, J., \& Clifford, C. W. (2005). Mechanisms selectively engaged in rivalry: Normal vision habituates, rivalrous vision primes. Vision Research, 45, 707-714.

Petrides, M. (2005). Lateral prefrontal cortex: Architectonic and functional organization. Philosophical Transactions of the Royal Society of London, Series B, Biological Sciences, 360, 781-795.
Pinkus, A., \& Pantle, A. (1997). Probing visual motion signals with a priming paradigm. Vision Research, 37, 541-552.

Rees, G., Kreiman, G., \& Koch, C. (2002). Neural correlates of consciousness in humans. Nature Reviews Neuroscience, 3, 261-270.

Sala, J. B., Rama, P., \& Courtney, S. M. (2003). Functional topography of a distributed neural system for spatial and nonspatial information maintenance in working memory. Neuropsychologia, 41, 341-356.

Scalaidhe, S. P., Wilson, F. A., \& Goldman-Rakic, P. S. (1999). Face-selective neurons during passive viewing and working memory performance of rhesus monkeys: Evidence for intrinsic specialization of neuronal coding. Cerebral Cortex, 9, 459-475

Sterzer, P., Russ, M. O., Preibisch, C., \& Kleinschmidt, A. (2002). Neural correlates of spontaneous direction reversals in ambiguous apparent visual motion. Neuroimage, 15, 908-916.

Tong, F., \& Engel, S. A. (2001). Interocular rivalry revealed in the human cortical blind-spot representation. Nature, 411, 195-199.

Tong, F., Nakayama, K., Vaughan, J. T., \& Kanwisher, N. (1998). Binocular rivalry and visual awareness in human extrastriate cortex. Neuron, 21, 753-759.

Wilson, H. R. (2003). Computational evidence for a rivalry hierarchy in vision. Proceedings of the National Academy of Sciences, U.S.A., 100, 14499-14503.

Wunderlich, K., Schneider, K. A., \& Kastner, S. (2005). Neural correlates of binocular rivalry in the human lateral geniculate nucleus. Nature Neuroscience, 8, 1595-1602. 